\title{
Cybercities: Mediated Public Open Spaces - A Matter of Interaction and Interfaces
}

\author{
Stefan Zedlacher ${ }^{1(凶)}$, Anna Khromova ${ }^{2}$, Eva Savina Malinverni ${ }^{2}$, \\ and Preben Hansen ${ }^{3}$ \\ ${ }^{1}$ Institute of Art History, University Graz, Graz, Austria \\ office@zedlacher.at \\ ${ }^{2}$ Facoltà di Ingegneria, Università Politecnica delle Marche, Ancona, Italy \\ khromova.anyu@gmail.com, e.s.malinverni@univpm.it \\ 3 Department of Computer and Systems Sciences (DSV), Stockholm University, \\ Stockholm, Sweden \\ preben@dsv.su.se
}

\begin{abstract}
In the near past, sources of information about public open spaces were: people, the place itself and historical archives. Accordingly, the information could be obtained by interviewing the visitors, by reading some poorly equipped signs on monuments or by research in libraries. Today, a new source appeared: The place itself covers its own information by the mean of the growing of the ICT (Information Communication Technologies). In addition, the information can be personalised in a way each people can access it individually. Ten years ago, a left-over newspaper on a park bench was a compact piece of information. Today, the newspaper resides on a smartphone in our pockets. In the future, the park bench will still be there, but dramatically changed to an IoT (Internet of things) object, bringing information to the people. Therefore, there is the need to re-think the park bench as an interface. A simple, fundamental point is: the quality of the interface rules the quality of the information. With a special focus on the latter, this chapter discusses how the classical model of the city is enhanced with the senseable city concept and how digital information influences, adopts, transforms and re-configures different objects in urban areas.
\end{abstract}

Keywords: Mediated spaces $\cdot$ Interfaces $\cdot$ Design methods $\cdot$ Senseable city

\section{Introduction}

In his 2002 motion picture Minority Report, based on the same name novel by Philip K. Dick from 1990, the director Steven Spielberg leads his main actor through a public open space (POS). In this 20-seconds scene, the actor gets, while escaping from prison, a lot of personalised advertising while the people around him are informed that he is a volatile criminal. The whole place with its infrastructure (screens, entrances, etc.) seems to be real-time responsive to the (fictional) situation - even to the operations elsewhere, shown in the scenes before and after. The phenomenon is the change of the POS to an innovative cyber one, in agreement to new concepts like smart city (Gibson et al. 1992; 
Hollands 2008). To answer the question "Which interactions is a POS in Cyber Cities capable for?", the analysis of city planning processes is a good starting point.

\section{Theoretical Framework}

In agreement with the vision shared by George Kubler in his book "The shape of time" the city design can be interpreted as a series of ideas in the history of human thinking, divided into four groups (Barnett 2016): Modern city design (Chandigarh), Traditional city design (Rome, Paris), Green city design (Surabaya) and System city design (Dubai). The main idea is that these listed concepts are not opposed to each other or mutually exclusive, rather they interplay with each other, being each one periodically more or less important (according to the duty of the project).

\subsection{The Intelligent City Concept}

The intelligent city concept is incorporated in the smart city, e.g. a city that is affected by technology, and also the self-organising city, which is without technologies but has a strong flexibility of the urban structure. The paradigm of smart city concept is based on interlacing the technological systems and human intelligence with the aim of better results and improved productivity. In the intelligent city concept, two types of approach can be identified: the top-down and the bottom-up. Both of them are aimed to support human needs by the mean of technology, but while the first one proposes solutions coming from the head of any organisation centre, the second one directly comes from the final users. Both types of systems have four components: Sensors, Collected data, Predictive models and Interfaces.

Here, a main difference between fiction and the real world arises: The fictional story from the introduction only takes the top-down city-concept in account. Talking about the top-down approach, the target is organising urban facilities in the cities to get better response for collective needs (energy management system, traffic control and incident management). The Bjork Ingals Group proposed to BMW to organise a competition having the title: The city of the futures (B. I. Group 2017). The winner of the project presented how people and cars can share space without frightening each other. The intelligence resides both in the vehicle and in the environment through sensors and lightning. Basically, the self-driving car informs the environment about its intentions, e.g. to sensors hidden in the pavement. Then, the latter creates a safe zone called area of influence for pedestrians, where no vehicles are allowed to enter. It has to be remarked how such a smart streets concept can make public open spaces safer for citizen. Passing to the bottom-up approach, it can be sufficient to integrate intelligent systems, that make the city perform better, instead of fundamentally rethinking the city design. Interfaces are the key component for this integration. Bottom-up examples like Copenhagen Wheel (Superpedestrian 2018) or Stuttgart's Luftdaten (2018) demonstrates that it is not necessary to adjust the city with new static sensors. Equipment that are already around (bicycles, clothes, baskets) could be used for data collecting process as a Mobile Sensors. Additionally, equipping bicycles with sensors effectively turns a city into an interface that collects data in real-time. The integration of such kind of bottom-up intelligence in the city and its surfaces could upgrade the quality of citizen life. 
The paradigm of Self-organising city concept performs a kind of urban play-ground where planners use techniques that don't impose a preconceived plan: the citizen freely share their ideas and projects with each other and, mostly important, with planners. The city is seen as an organism that doesn't need to follow any human master plan for growing and functioning. The role of the planners is to guide the existing self-organising concepts in the urban environment. Tracing back this concept to the idea of Metabolism in the '60s, a Japanese group of architects (e.g. Kisho Kurokawa's Helix City Project) fused ideas about architectural mega-structures with those of organic biological growth (taking inspiration from the recently discovered DNA). Continuing the chronological time-line, the Hungarian architect Yona Friedman made his contribution in designing a mobile architecture that he called a Spatial Infrastructure (Friedman 1956). In that experimental project the user plays a key role in the city design process. The citizen was allowed to freely use the mobile city's structural system according to his needs and wishes, which is perfectly in line with the self-organising city concept. In the same period the rising of cybernetics in the field of communication and technology impacted on the architects' methods and visions (Pask 1969). The main concept is based on the notion that intelligence lies in its capacity to learn from feedbacks. That new vision shifts the role of planner in the design process and opens the necessity to bring new technologies that can help machines to react to a real-time stimulus. By applying this concept to the planning process, an interesting question arises: Could the cities and their buildings learn from their usage and auto-correct themselves into an optimal configuration and anticipating future uses? Also, computer programmes as the SimCity series games (Jonathan Burnett) or the MVRDV Function-mixer (Hartog 2006) shown a good example of how technologies bring new visions in urban planning. The virtual simulation of urban environment explores the use of computer-generated scenarios for having a support in taking decisions and planning solutions. This involvement helps people, municipalities and communities to find optimal configurations. But, projects from the intelligent (smart) concepts are mainly top-down and ignoring the existence of interfaces at all. Although they have sensors, predictive models and collected data which are also parts of the fictional city concept from Minority Report.

\subsection{The Cyborg-Society and the Senseable City Concept}

"Permit man's existence in environments which differ radically from those provided by nature as we know it" (Clynes and Kline 1960: 29-33) was the starting point for rethinking the concept of the human body and its extensions and possibilities. This new way of thinking gave the perspective for the first cyborg project: "cyborg is a hybrid capable of more than either the biological or the mechanical system alone can do, with a correspondingly expanded range of possible habitation" (Ratti and Claudel 2016: 42-49). The tools and all innovations people have been aware, allowing humanity to survive in extreme conditions that they otherwise could never adapt. Serving as an additional option to the biological presence defines us as a kind of cyborg. "Human progress was marked by the gradual externalization offunctions, from stone knives and axes that extended the capacity of the hand to the externalization of mental functions with the computer." (Picon 2004: 114-121). If in the past the innovations were created as an enhancement of the physical body, today they are mainly used as an extension of 
the possibilities of the mind: "... digital technologies have become a dynamic extension of our bodies and minds, demanding a constant and two-way cybernetic exchange in a way that our traditional (one-way) extensions, such as clothing or axes, have never done" (Ratti and Claudel 2016: 42-49). Interesting aspects appeared, as the deep natural involvement of technological system into human environment. "We [...] are provided with two types of bodies ... the real body which is linked with the real world by means offluids running inside, and the virtual body linked with the world by the flow of electrons" (Ito 1997: 132). Smartphones became the strongest connection between the two bodies and so recognised as an interface. It additionally extends humans memory and logical capacities in such a marked way that the person is not considered a simple human anymore but kind of an upgraded version of it: a post-human. "A new entity that is born with technology rather than acquiring it ... where each individual's mental and social existence is enable, sustained and improved by technologies." (Ratti and Claudel 2016: 42-49).

The main technology mentioned are the so-called smart-devices. They are changing the way people think about a city and its infrastructure in a radical way: thanks to the rising of digital networks what before was passive is now active, so every aspect of an urban reality should now be able to be interfaced with smartphones. In simple words, a modern city should give the post-humans an environment suitable for them. With the rising of smart-devices still both methods from the intelligent city in the senseable city concepts, could be found. But with different notion:

- Tracking as the top-down method where the information is gathered and visualized for decision-makers, stakeholder, city council or researcher (Ratti et al. 2007).

- Interaction as the bottom-up example with, e.g. QR codes (Foth et al. 2015). The interaction includes human to human, human to companies (marketing) or authorities (information) or human to machine while the layer that carries the QR code is differently (i.e. cloths, streets, buildings, etc.)

The senseable city concept pushes further the interactivity of this innovative way of thinking about POS. In a short period, places became a breathing, living entity acting on a large-scale with the inhabitants (what we previously defined as cyborgs). The latter will create, plug into and interact with this new entity, and so, digital spaces will be naturally interacted and above all spontaneously born onto POS. This reality was, among others, mentioned and explained by Mark Weiser, ac-cording to which the interfaces would find no places anymore since everything will be intimately absorbed into the city. Allowing then a complete merging between the digital and the physical spaces a "new way of thinking about computers in the world, one that takes into account the natural human environment and allows the computers themselves to vanish into the background." (Weiser 1991: 1). Another realisation of the senseable city concept can be found in the socalled boards, e.g. interactive screens disseminated all along the cities (Fig. 1) where POS infrastructure are transformed into intercommunicating interfaces, away from smartphones. Basically, these new generation billboards are in effect web browser applications capable of all the tracking and advertising functionalities known from any desktop website. So, what was oriented towards a single user before is now becoming public: a desktop browser turns to a public display where advertises take place, all integrated into the POS. This scene of the 2002's Minority Report became reality. 
In summary, the senseable city concept can be resumed as the "Technology recedes into the background, and interaction is brought to the fore. Buildings and public open spaces can be simple-rather than voluptuous and shocking - but even more integrally vibrant and living" (Ratti and Claudel 2016: 62-73).

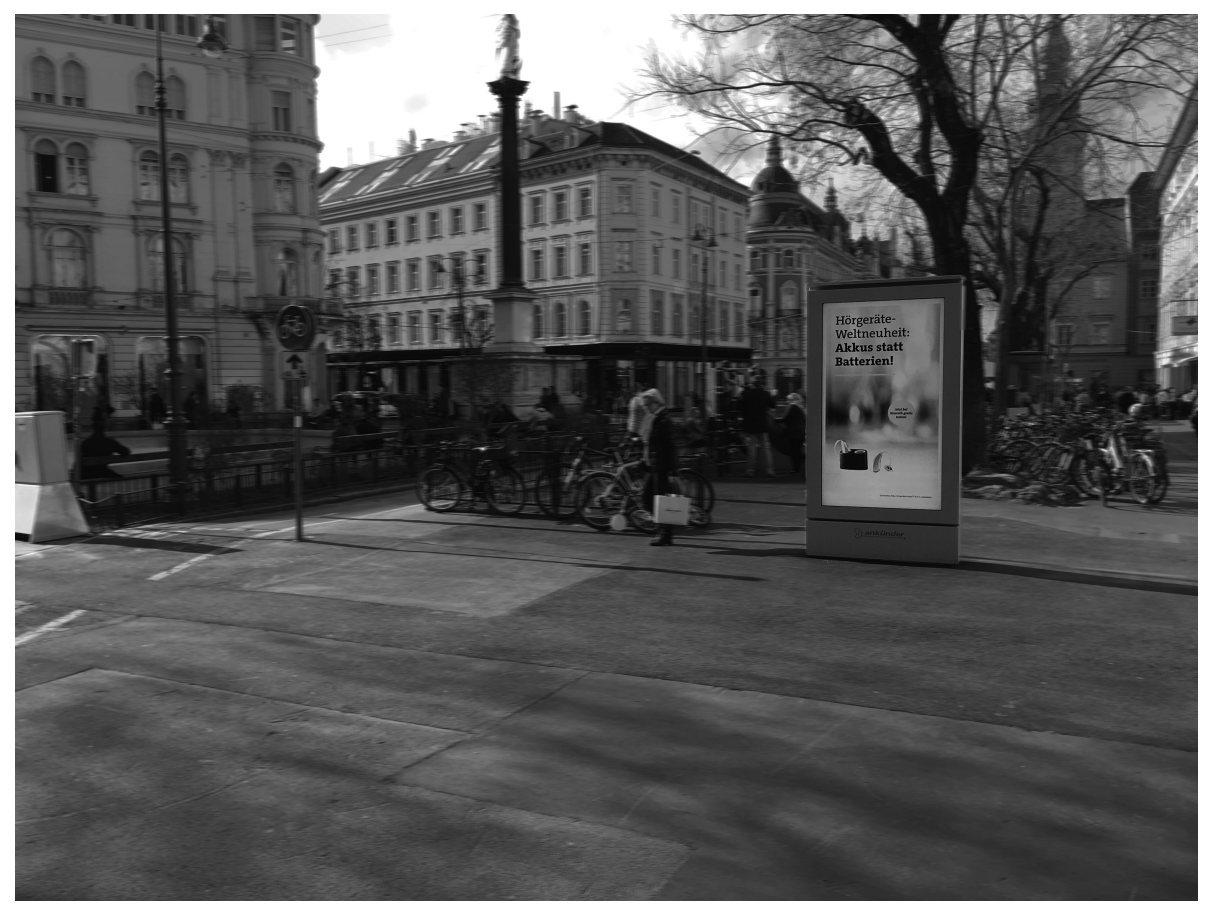

Fig. 1. Graz city information terminals (Source: authors. 2017)

\section{Interaction Design and Interfaces}

Although, as widely discussed in the previous sections, the city of the future completely neglects the necessity of having an interface between citizen and spaces (since the digital and the physical domains are perfectly interlaced between each other), nowadays they still are of fundamental importance since the progress is still making the first steps toward such reality. A brief overview of the basic concepts of interactions and of the various kinds of interfaces will been given in the present section.

\subsection{Basic Concepts of Interaction}

One of the aims of interaction design is to create an interface that makes efficient and user-friendly for a user to interact, operate and manage a digital or physical electronic device. Basically, this means that there should be some kind of input to achieve the desired output. Within interaction, there are several crucial and important concepts that 
need to be considered in a design process, in which the interaction between a human and a device (physical or digital) is a vital component. Interaction and design for interaction in physical or blended (Benyon 2014: 420-425) POS may also be viewed and understood as explicit interaction or implicit interaction. Explicit interaction usually builds on the classical desktop screen-based interaction metaphor. This often result in that the human need to cope with different technological issues in order to achieve a certain goal. In contrast, implicit interaction design puts the human in the foreground and the technology in the background. These are important aspects of interaction in (blended) mediated spaces since that may have the possibility to entangle and embed into our daily activities in both urban and rural environments. Some important concepts are that may apply to design of POS, mediated or not:

- Dynamics - This concept refers to indications of current and changing modes and states and how a device, components or system adapts to different contexts, situations, tasks, people etc. (Buxton 2010; Löwgren and Stolterman 2004).

- Temporality - It refers to the concept of time. In design it can concern, for example, the extension over time, the duration of an interaction, the immediacy of the interaction activity, the delays and pauses within an interaction, the segmentations of an interaction, the separation and connection of several interaction sequences, the pace and rhythm of an interaction, and the periodicity (Buxton 2010; Löwgren and Stolterman 2004).

- Interactivity - The interactivity generally refers to how users and (digital and physical) artefacts initiate and respond to different actions including action openings, turn-taking by humans but also by tasks of a device, and closures (Löwgren and Stolterman 2004).

- Sequentiality - One very important aspect of designing for interaction refers to how interactions are ordered and structured; the flow of different actions and tasks to be accomplished, how courses of events are planned and how much freedom, control, guidance, support etc. are built into activities with the service, components or system (Benyon 2014; Löwgren 2002).

- Context of use - This concept refers to placing the planned activities, objects, services or system in specific social and physical contexts which exemplify the artefact in use (Gedenryd 1998; Ozenc et al. 2010; Suchman 1985).

Different interaction design qualities are defined by Lim et al. (2007: 246) as something that appears only in (inter)action: "The interaction gestalt also has to be designed in a way that will evoke the desired user experiences. The designer has to anticipate how a certain gestalt will be experienced by a user, and that anticipation has to be translated back into ideas on how the gestalt should be shaped". In interaction design in general, Lim et al. (2007) define different qualities that may apply for the context and situation in which people interact in POS. The authors also talk about the "...the interaction gestalt..." that it need to be considered and approached in the design process as well. The "gestalt" is very important since it is to evoke the desired user experience. This means that the designer needs to anticipate how users in a POS can experience a certain gestalt. So, when designing for interactions, this anticipation needs to be translated back into ideas on how a "gestalt" should be shaped and formed. Löwgren (2002) proposes to think about interaction design in terms of specific use 
qualities. Use qualities are certain properties of a digital design that are experienced in its use situation or context. According to the author, such qualities transcend the specific design. At the same time, it offers a certain language to be used when talking about design. Furthermore, the author suggests a set of qualities that design of public open spaces needs to consider: motivation, interaction per se, social aspects of the object or systems, structural qualities, meaning-making.

\subsection{Types of Interfaces}

The role of a user interface is to give to the final user a dedicated platform to control generic software (that, in turn, has been defined on hardware). Although in the recent past the concept of interface was limited to the engineering world, nowadays, thanks to the use of sensors in multiple fields (applications in medicine, architecture, landscape planning etc.), it has to be used and understood in a broader meaning. The way users interact with interfaces depends on the interface in use. While the most common one has been for decades the simple textual command line interface, nowadays a huge variety of interfaces are available, where all the senses of the human body have been exploited. Generally speaking, the available kinds of interfaces are:

- Voice User Interface (VUI) - In this first kind of interface, the human voice is the vehicle through which the information is carried from the user to the software. Various challenges are currently being addressed in the developing of such interfaces, all more or less related to the voice recognition issue, that allows a more functional voice-controlled interface. In turn, the interaction re-quired with this kind of interface is quite natural since it doesn't require any-thing else than the vocal command of the user.

- Tangible User Interface (TUI) - As the name suggests, this kind of interface is based on a physical medium that stands between the user and the software. The latter can be a door, a spoon, or in general an available object in an urban space. Although the TUIs are relatively new, numerous examples can be found in museums, architecture (Wang et al. 2017a), furniture and clothing, and this remarks the importance of such a kind of interface. An interesting aspect regards the experience that the user gets from the just introduced interface: the more playful and active way of interacting facilitates a more creative and learning effect (Wang et al. 2017b).

- Natural User Interface (NUI) - While a TUI exploits tangible objects to create a way of communication between the software and the user, a NUI reaches the same result making use of gesture ( $\mathrm{Li}$ et al. 2016) and eye-interaction. This results in a more natural way of interacting.

- Brain Computer Interface (BCI) - Finding its place in the borderline of the scientific progress, the BCI is probably the most interesting and appealing kind of interface that can be found today. The interaction between the user and the software is here carried out by the mean of electric impulses running through the brain and collected by the mean of electrodes. The BCI will make possible to control robotic arms, machines etc. in a large variety of application areas (medical, industrial, etc.). 
Such kind of innovation will, in addition, help many people with disabilities in improving their life in a huge number of situations.

- Cross-Object User Interfaces (COUI) - Cross-objects user interfaces is a literally new term that describes user interfaces spatially distributed across object surfaces in the physical world and the virtual reality in a similar way. In contrast to tangible user interfaces, COUIs are inhabited in physical or virtual objects in flexible ways and are compatible with multiple and different interaction methods (Sun et al. 2018; Li et al. 2017).

Starting with the smartphone in the previous chapter we hopefully verified with this chapter the huge variety of possible interfaces for the POS. In the next chapter we combine the city design methods mentioned, the types of interfaces and the evolution of POS to a possible explanation.

\section{The Ternary Structure of a POS and Its Scientific Rationalities Interpretation}

According to the city design methods previously explained, the reality of a POS has changed over the last decades. They are not interpreted as physical spaces, but rather as entities that embrace different dimensions into the same system. In fact, according to the vision of Stanley Milgram (Milgram et al. 1992), space is made not only of physical characteristics, but it has also a mental image that lives in human mind. One step further in Philip K. Dick's vision from Minority Report, it is a visual, digital representation of an individual view on a common base (the POS).

A simple yet effective way to describe POS is the ternary system of Nature, Culture and Geist (Khromova et al. 2016), where:

- Nature is the environment in its essential form, i.e. without any human interference.

- Culture is any expression of human's creativity, that can be differentiated into tangible (materials and artefacts) and intangible (values, traditions, beliefs, etc.).

- Geist is the source of human's creativity itself; it defines human's goals, objectives and lines of action that modify the nature towards the realization of what we defined culture (Fig. 2).

To understand this complex approach of POS, the three-different kind of scientific rationalities (Stepin 2008) will be introduced: the classical, non-classical and post-nonclassical. The first kind of scientific rationality, the classical rationality, deals with what we may call simple systems, where all properties of its parts define the whole system. They are delimited against external components like space and time and their elements are causal deterministic. Such systems are essentially a mechanical combination of their components, whose interactions are completely neglected. Essentially, "the classic rationality approach analyses it by simply considering the properties of its different sections for then adding them up together to get the global overview of the set" (Khromova 2018: 28-33). For example, McLuhan's (1964) vision of a social system made only of unidirectional interaction between the mass of people and the source of 


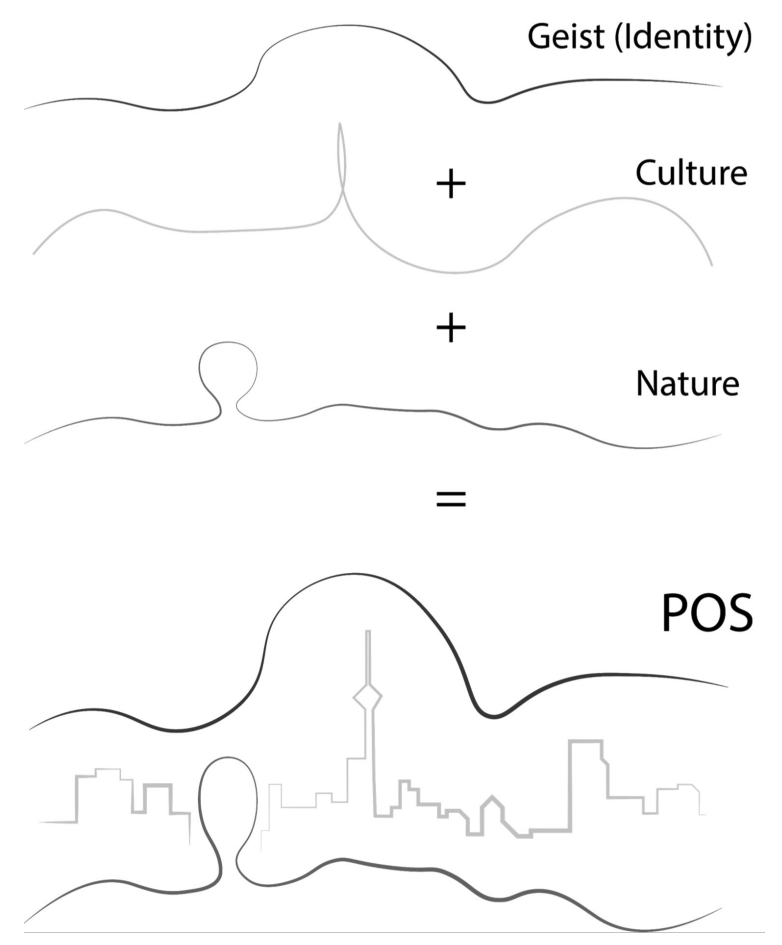

Fig. 2. The public spaces ternary structure: Geist, Culture and Nature (Source: authors)

information (as television or radio) is efficiently described by the mean of the just introduced first kind of rationality.

When dealing with large systems including autonomous subsystem having a huge number of interactions between each other, the classical rationality approach becomes obsolete. It leads to the second kind of scientific rationality, the non-classical. With an integrated "control-block" the system has a build in feed-back loop for its elements and subsystems. The feedback of each element runs into a simple programme whose output determines the behaviour of the system. This ongoing reproduction of organised elements could be found in society (social objects), nature (organisms) or populations of any kind. The whole is more than its individual parts. A multicellular organism provides one of the best examples of a right application of the non-classical rationality. Nowadays design of POS is strongly related to the non-classical rationality concepts since the aim in creating harmony by considering all the components of the POS itself (Khromova 2018).

The last kind of scientific rationality, defined as post-non-classical, relates to systems evolving themselves through self-regulating. This kind of system is "characterized by the development that leads from one self-regulation type to another. Each newly developed level causes back effects on earlier formed ones and restructures them, the system thus acquires a new integrity. The system changes as new levels emerge, with new relatively autonomous subsystems being formed there. Openness, exchange of 
energy and information with the surrounding are key attributes of complex, selfdeveloping systems. A modern city with its services exemplifies it. The self-regulating aspect is represented by the huge variety of different agencies such as the communal office, the land registry office, services for the city, etc., whose usefulness would vanish without a proper interaction with all other public authorities such as health services, police stations, etc. The post-non-classical rationality finds its applications in many recent projects aimed to update the city. One of the best examples is given by the Futurecraft project (Ratti and Claudel 2016), where planners don't provide anymore a fixed, pre-conceived and so strict indication about what their vision of the city-design of the future will be. Basically, conditions, scenarios and consequences are shared and discussed. This method supports public debates and spreads ideas and alternatives where people construct new values and guides to POS design. By turning classical design of urban space upside-down, planners (not exclusively) called this approach mutations. Such mutations will then grow, evolve and finally end up in creating tangible artefacts (pre-interfaces) in the reality of the city. An example implementing the post-non-classical method is the Trash Track project from MIT senseable city lab, "where cheap tags equipped with GPS localization are applied to rubbish and a fullscale urban demonstration was created to test it. As a result, subsequent discussion and debate has led to systemic improvements by waste management companies, startup companies that produce trash trackers, and, most importantly, citizens where inspired to reduce waste and to recycle" (Ratti and Claudel 2016). Switching back to our topic of interest, a synergetic urban landscape like a POS equipped with ICT may be represented by the post-non-classical scientific rationality.

One should also notice that, according to what has been said and to the examples that has been given, the three proposed approaches for describing and interpreting a generic system don't abolish nor replace each other. Contrarily to what one can think, the post-non-classical vision, although clearly more complete in respect to the classic one, is not always the best choice since sometimes its complexity can be completely avoided, resulting in a much immediate and practical interpretation of the system under investigation.

\section{Conclusions}

With the ternary system of public spaces, the co-existence of bottom-up and top-down methods in senseable city systems are proven. Which one of the scientific rationalities (between the classic, non-classic and post-non-classic) is more suitable to a POS equipped with ICT strongly depends on the attitude of the planner. This fact also remarks how each new type of rationality doesn't imply the rejection of the previous one, but only completes our set of tools for analysing any kind of system, from the simplest ones (where the classic kind of rationality is preferred) to the more complex ones (where the post-non-classical is often the right choice). The tools could be part of the interfaces.

Currently, interfaces that support tracking and interaction are not reduced to smartphone. Rather they harness IoT and possibly change to tools for analysing and interaction. We have also shown that the interfaces, beside mobile devices, are deeply 
underdeveloped in their usage for a cyberpark, compared with their possibilities. But, comparing Mark Weiser's vision for computer[s] for the 21st century (Weiser 1991), the have developed further. The way disseminating information from the cyberspace to the user, i.e. the interfaces and their different types, is being a real-time connection between city-planner and all the other components of the cyber-area. The role of cityplanners (and their computers!) in a senseable city has to be redefined.

Further work has also to be done in order to deeply investigate what may be the weak points of the conception of cyberparks, such as the growing information pollution and peoples experience with the (digital) urban environment. According to that, the political structures and decision-making process must be updated to a real-time information system. Therefore, additional efforts should also be put in realizing a structure where individuals can choose how much they want to be involved in the cyber dimension, according their needs and comfort. The ground level for this structure could be the total absence of the cyber interaction and a balanced synergy between personal will and pre-built scenarios. Realtime, reactive POS, by the way described in the beginning, are possible, but nevertheless they lack the connection between their users, their inherent systematics and the quality of interfaces. Minority Report with the vision of Philip K. Dick is only a shadow of today's capabilities.

\section{References}

B. I. Group, 17 September 2017. http://audi-urban-future-initiative.com/blog/bjarke-ingels-group. Accessed 21 Aug 2017

Barnett, J.: City Design: Modernist, Traditional, Green and Systems Perspectives. Routledge, New York (2016)

Benyon, D.: Designing Interactive Systems: A Comprehensive Guide to HCI and Inter-Action Design. Addison Wesley, Harlow (2014)

Buxton, B.: Sketching User Experiences: Getting the Design Right and the Right Design. Morgan Kaufmann, San Francisco (2010)

Clynes, M.E., Kline, N.S.: Cyborgs and space. Astronautics 14(9), 26-27 (1960)

Foth, M., Brynskov, M., Ojala, T.: Citizen's Right to the Digital City: Urban Interfaces, Activism, and Placemaking. Springer, Singapore (2015). https://doi.org/10.1007/978-981287-919-6

Friedman, Y.: (1956). http://www.yonafriedman.nl/?page_id=225. Accessed 13 May 2018

Gedenryd, H.: How Designers Work - Making Sense of Authentic Cognitive Activities. Cognitive Science, Lund (1998)

Gibson, D.V., Kozmetsky, G., Raymond, W., Smilor, R.W.: Smart Cities, Fast Systems, Global Networks. The Technopolis Phenomenon. Rowman \& Littlefield, Lanham (1992)

Hollands, R.G.: Will the real smart city please stand up? City 12(3), 303-320 (2008)

Hartog de, H.: MVRDVs 3D urban design, 10 November 2006. https://www.archined.nl/2006/ 10/mvrdv\%C2\%92s-3d-urban-design. Accessed 3 May 2017

Ito, T.: Tarzans in the media forest. In: 2G, no. 2, p. 132 (1997)

Khromova, A., et al.: Is the mediated public open space a smart place? Relationships between urban landscapes and ICT, The Cost Action TU 1306 CyberParks. SCIRES-IT (SCIentific RESearch) and Information Technology, pp. 17-28 (2016) 
Khromova, A.: Are the Urban Parks becoming Cyberparks? The Developing of Public Open Spaces: ICT tools to support the landscape planning process (Unpublished thesis) UNIVPM (2018)

Li, X.A., Hansen, P., Lou, X., Geng, W., Peng, R.: Design and evaluation of cross-objects user interface for whiteboard interaction. In: Streitz, N., Markopoulos, P. (eds.) DAPI 2017. LNCS, vol. 10291, pp. 180-191. Springer, Cham (2017). https://doi.org/10.1007/978-3-31958697-7_13

Li, X.A., Lou, X., Hansen, P., Peng, R.: On the influence of distance in the interaction with large displays. IEEE J. Disp. Technol. 12(8), 840-850 (2016)

Lim, Y., Stolterman, E., Jung, H., Donaldson, J.: Interaction gestalt and the design of aesthetic interactions. In: Proceedings of the 3rd Conference on Designing Pleasurable Products and Interfaces, pp 239-254. ACM Press, New York (2007)

Löwgren, J., Stolterman, E.: Thoughtful Interaction Design - A Design Perspective on Information Technology. The MIT Press, Cambridge (2004)

Löwgren, J.: The use qualities of digital designs. complete draft 1.0, 21 October 2002. http:// citeseerx.ist.psu.edu/viewdoc/download?doi=10.1.1.196.4873\&rep=rep1\&type=pdf. Accessed 13 June 2018

Luftdaten, 12 January 2018. https://luftdaten.info. Accessed 23 May 2018

McLuhan, M.: Understanding Media: The Extensions of Man. McGraw-Hill Book Company, New York (1964)

Milgram, S., Sabini, J.E., Silver, M.E.: The Individual in a Social World: Essays and Experiments. McGraw-Hill Book Company, New York (1992)

Ozenc, F.K., Kim, M., Zimmermann, J., Oney, S., Myers, B.: How to support designers in getting hold of the immaterial material of software. In: Proceedings of the 28th Annual CHI Conference on Human Factors in Computing Systems, Atlanta, Georgia, 10-15 April, pp. 2513-2522 (2010)

Pask, G.: The architectural relevance of cybernetics. Architectural Design, September issue No 7/6, 494-496 (1969)

Picon, A.: Architecture and the virtual: towards a new materiality? Praxis 6, 114-121 (2004)

Ratti, C., Claudel, M.: The City of Tomorrow: Sensors, Networks, Hackers, and the Future of Urban Life. Yale University Press, New Haven (2016)

Ratti, C., Sevtsuk, A., Huang, S., Pailer, R.: Mobile landscapes: graz in real time. In: Gartner, G., Cartwright, W., Peterson, M.P. (eds.) Location Based Services and TeleCartography. Lecture Notes in Geoinformation and Cartography, pp. 433-444. Springer, Heidelberg (2007). https:// doi.org/10.1007/978-3-540-36728-4_31

Stepin, V.S.: Types of systems and types of scientific rationality. SATS 9, 27-43 (2008)

Suchman, L.A.: Plans and Situated Actions - The Problem of Human Machine Communication. Xerox, Palo Alto (1985)

Sun, L., Zhou, Y., Hansen, P., Geng, W., Li, X.: Cross-objects user interfaces for video interaction in virtual reality museum context. Int. J. Multimedia Tools Appl. May 2018 (2018, forthcoming). https://doi.org/10.1007/s11042-018-6091-5

Superpedestrian, 21 March 2018. https://www.superpedestrian.com/tech. Accessed 5 June 2018

Wang, Y., Luo, S., Liu, S., Lu, Y., Hansen, P.: Crafting concrete as a material for enhancing meaningful interactions. In: Kurosu, M. (ed.) HCI 2017. LNCS, vol. 10271, pp. 634-644. Springer, Cham (2017a). https://doi.org/10.1007/978-3-319-58071-5_48

Wang, Y., et al.: AnimSkin: fabricating epidermis with interactive, functional and aesthetic color animation. In: Mival, O., Smyth, M., Dalsgaards, P. (eds). Proceedings of the 2017 Conference on Designing Interactive systems, Edinburgh, UK, pp. 397-401 (2017b)

Weiser, M.: The computer for the 21st century. Sci. Am. 265(3), 94-104 (1991) 
Open Access This chapter is licensed under the terms of the Creative Commons Attribution 4.0 International License (http://creativecommons.org/licenses/by/4.0/), which permits use, sharing, adaptation, distribution and reproduction in any medium or format, as long as you give appropriate credit to the original author(s) and the source, provide a link to the Creative Commons license and indicate if changes were made.

The images or other third party material in this chapter are included in the chapter's Creative Commons license, unless indicated otherwise in a credit line to the material. If material is not included in the chapter's Creative Commons license and your intended use is not permitted by statutory regulation or exceeds the permitted use, you will need to obtain permission directly from the copyright holder. 